\title{
Variations of the human retinal vascular patterns: A descriptive cross- sectional study in Sri Lanka
}

\author{
Sanjayan $\mathrm{A}^{1}$, Ruwanpathirana $\mathrm{PS}^{1}$, Dissanayake $\mathrm{MM}^{1}$ \\ ${ }^{\prime}$ Department of Anatomy, Faculty of Medicine, University of Colombo. \\ *sanji022@yahoo.com
}

The retina is the commonest site used to observe the vascular architecture invivo. Changes in retinal vasculature have been described in numerous medical conditions; detection of which can aid diagnosis. However, variations of the normal vascular pattern have not been studied extensively. Fundoscopic images $(\mathrm{n}=150)$ of normal individuals were manually evaluated qualitatively for distribution patterns of main vessels. Images with retinal, vascular pathologies were excluded by a consultant ophthalmologist. Of the 104 images $67.3 \%(n=70)$ were of females and $49 \%(n=51)$ were from the left eye. Standard pattern of 2 nasal and 2 temporal were seen in $89.4 \%(\mathrm{n}=93)$. Six other different vascular patterns were seen. Distribution of the temporal vessels was constant. Three eyes

(2.9\%) from 2 individuals had only one nasal branch (transverse). Both eyes from one person $(1.9 \%)$ had a transverse nasal branch in addition to two upper and lower nasal branches. Four eyes (3.8\%) of three people had two upper nasal branches of which three eyes ( 2 from the same person) had one lower nasal branch and the other eye had 2 lower nasal branches. There was one $(0.9 \%)$ eye with three upper nasal branches and one $(0.9 \%)$ lower nasal branch and one eye with one upper nasal branch and two lower nasal branches. Although standard pattern of vascular distribution was seen in majority, variations were seen in the nasal branches. These findings can be used for development of digital technology in detecting retinal and vascular diseases involving angiogenesis. 